\title{
Margin Uninvolved by Invasive Melanoma
}

National Cancer Institute

\section{Source}

National Cancer Institute. Margin Uninvolved by Invasive Melanoma. NCI Thesaurus.

Code C159409.

A result that the marg in is uninvolved by invasive melanoma. 